\title{
Discretized Air Phase Modeling in the Simulation of Pipeline Refilling Operations
}

\author{
Jose G. Vasconcelos and Bernardo C. Trindade
}

\subsection{Introduction}

This chapter focuses in the numerical simulation of the filling of water mains, and how to incorporate the effects of air phase in those computations. Water mains are part of an aging urban infrastructure, and maintenance procedures are becoming more frequent; some maintenance procedures require the emptying and subsequent refilling of pipelines. Water main refilling is also observed in water main commissioning or following power outages. In any condition, the refilling process is performed very cautiously due to the possible interactions between water and the air initially present in the pipes, as explained below. Because of the gradualness, pipeline refilling may be a time consuming process, which may adversely affect water users.

During the filling process, at a certain inflow rate one cannot exactly predict whether, or at what locations, air pockets will form. If such pockets are compressed during the filling events, severe pressure surges may appear. On the other hand, if the filling cannot eliminate all the air pockets within the pipeline, other issues can arise as air pockets may block the flow, create additional energy losses, reduce pipe conveyance or increase pumping electricity demand.

The problem of air pockets in water mains has raised the interest of different researchers, for example with regard to the development of a criterion of hydraulic removal of air pockets by the water flow. Some of these investigations were directed towards the development of numerical models to simulate the

Vasconcelos, J. and B.C. Trindade. 2012. "Discretized Air Phase Modeling in the Simulation of Pipeline Refilling Operations." Journal of Water Management Modeling R245-03. doi: 10.14796/JWMM.R245-03.

(C) CHI 2012 www.chijournal.org ISSN: 2292-6062 (Formerly in On Modeling Urban Water Systems. ISBN: 978-0-9808853-7-8) 
filling process in water mains. However, as it will be shown, there is a knowledge gap in terms of what is the most appropriate way to incorporate air phase pressurization in the water filling modeling.

Current investigation at Auburn University aims to address this gap and propose an improved numerical modeling tool, designed for water works to simulate the refilling of water mains. The final goal is to transform the filling process into an engineered and predictable process, decreasing the uncertainties associated with such events and possibly reducing downtime in pipelines caused by lengthy refilling.

\subsection{Literature Review}

Examples of pipeline filling models include the works of Liou and Hunt (1996), Izquierdo et al. (1999), Vasconcelos (2007) and Leite and Vasconcelos (2011), which are discussed below.

Possibly the first model for the simulation of sloped water main filling was proposed by Liou and Hunt (1996). It applied to the filling of long water mains with variations in topographic profiles. According to the authors, the model is applicable when the flow velocity $V$ is high enough to prevent the intrusion of air into the inflow front $\left(V>0.54(g . D)^{0.5}\right)$, where $g$ is the acceleration due to gravity and $D$ is the pipe diameter. The model assumed ideal air ventilation (air phase remains at atmospheric pressure) and further assumed that the inflow front remains well defined and vertical, resembling a water piston.

The main limitation of this model approach is the assumption of a well defined piston-like inflow front. This assumption may not be a good representation of the actual shape of the inflow front. Guizani et al. (2006) conducted several laboratory experiments on the filling of water pipelines with different inflow conditions and slopes, and in all cases the filling front shape resembled a wedge, similar to a dam break front propagating within the pipe. The authors point out that a vertical interface would be a valid assumption only if the flow rate within the pipe is very large.

Another modeling approach to the filling of sloped pipelines was proposed by Izquierdo et al. (1999). The goal was to simulate the filling assuming water accumulation in the lower points of the pipelines, leading to the creation of air pockets at each isolated high point. Upon flow start, air pockets were highly compressed by the surrounding water columns; the authors confirmed that smaller entrapped air pockets lead to larger pressure peaks, as was pointed out by Martin (1976). This model assumes that the transition interface between phases remains well defined and that the entrapped air does not intrude on the water advance. While this approach introduces an improvement over Liou and Hunt's approach, neither the initial water accumulation in lower points nor the 
sudden flow start are anticipated in water main filling processes. Moreover, this model neglects ventilation in the water main, which is a limitation since air valves are commonly found in water mains, and it assumes well defined airwater interfaces.

These two modeling approaches apply the lumped inertia approach to simulation of the water flow, which was initially conceived to simulate pressurized, gradually varying unsteady flows. The interface between air and water in the inflow front advancing from the supply reservoir is essentially the length of the rigid column so that difficulties in simulating air-water interfaces are avoided, but at the cost of introducing an unrealistic assumption. One alternative, to compute these interfaces and still have calculations within the realm of onedimensional models, is to rely in flow regime transition models, also referred to as mixed flow models. Two modeling alternatives have recently been proposed by Vasconcelos (2007) and Leite and Vasconcelos (2011) using mixed flow models.

Vasconcelos (2007) proposed a model that has the ability to simulate unsteady flow in both regimes (pipes pressurized or at atmospheric pressure). The model was implemented using the finite volume method to solve an adaptation of the Saint-Venant equations, which were initially proposed to model the transient flow of rivers and channels. Conceptual models such as the Preissmann slot (Cunge et al., 1980) and the two component pressure approach (TPA) (Vasconcelos et al., 2006) expand the applicability of these equations to simulate pressurized flow conditions in closed conduits.

Because this model did not make any assumptions regarding the shape of the air-water fronts, it explicitly calculates air-water interfaces, offering a better prediction of entrapped air pockets location during the filling events. The limitation of the Vasconcelos (2007) model is that it does not consider the effects of air pressurization, assuming an ideal ventilation system. This assumption is not realistic as ventilation is limited and air pressurization may affect the system dynamics.

The work presented by Vasconcelos et al. (2009) compared field measurements of water main filling with the predictions by Vasconcelos' (2007) model. Results were generally well predicted but discrepancies were noticeable and attributed to the inability of the modeling approach to simulate air pressurization. The computation time of these filling events was also considered high (often over two or three hours using a desktop computer).

Leite and Vasconcelos (2011) use a theoretically simpler approach to create a mixed flow model framework. Following the strategy of defining different flow conditions over time during the flow, the free surface flow portion was simulated with the kinematic wave equation, with the pressurized flow region using the rigid column approach, and the interface tracking was based on the 
continuity principle. It was assumed that a backward moving inflow front was gradual (without a hydraulic jump) and that air pressurization was uniform in the entire air pocket and could be calculated by using the ideal gas law and an orifice-type expression to relate pressure and air discharge. This modeling alternative still needs to be validated with experimental results.

In this present work, approaches that assume air pressure uniformity over the extent of the air pocket are referred to as a lumped air approach. This approach has been applied in different hydraulic models to simulate air-water interactions (e.g. Li and McCorquodale, 1999; Zhou et al., 2002; Vasconcelos and Wright, 2009; Leon et al., 2009). However, one relevant question concerns what is the applicability when an air pocket is much longer (as anticipated in sloped water mains), and when are friction energy losses in the air flow relevant? This study aims to explore these questions in more detail.

\subsection{Objective}

The objective of this work is to compare two modeling alternatives for the air phase in the simulation of the water main filling problem. The first alternative is the lumped air approach, which assumes the uniformity of air characteristics throughout; the second alternative performs a discretization on the air phase and simulates the flow by applying the Euler equations. Another objective is to study the general behaviour of the filling process in water main with a simple geometry.

In both of the proposed models, water phase is simulated applying the SaintVenant equations modified by the TPA model, similar to the strategy presented by Vasconcelos and Wright (2009), but including some improvements on the numerical modeling techniques proposed by Sanders and Bradford (2011).

\subsection{Methodology}

\subsubsection{Mathematical Model}

The mathematical model which is the basis for the proposed approach is a modified version of the Saint-Venant equations which is able to simulate flow regime transition between free surface and pressurized flow regimes.

Vasconcelos and Wright's (2009) model incorporates the two component pressure approach (Vasconcelos et al., 2006) and is further modified to incorporate air phase pressurization. Equations 3.1 and 3.2 present the water phase model in conservative divergent form: 


$$
\begin{aligned}
& \frac{\partial \dot{U}_{w}}{\partial t}+\frac{\partial \vec{F}_{w}}{\partial x}=S_{w}
\end{aligned}
$$

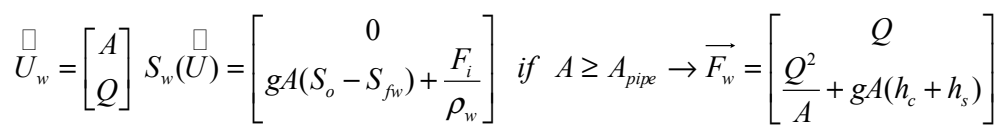

$$
\begin{aligned}
& \text { if } A<A_{\text {pipe }} \rightarrow \overrightarrow{F_{w}}=\left[\begin{array}{c}
Q \\
\frac{Q^{2}}{A}+g A\left(h_{c}+h_{s}\right)+g A_{\text {pipe }} H_{\text {air }}
\end{array}\right]
\end{aligned}
$$

where:

$$
\begin{aligned}
A= & \text { cross sectional area of the water flow, } \\
h_{c}= & \text { depth of the centroid of the flow cross section, } \\
h_{s}= & \text { surcharge head occurring only in the pressurized flow } \\
& \text { regime, } \\
A_{\text {pipe }}= & \text { cross sectional area of the conduit, } \\
B= & \text { width of the free surface flow, } \\
S_{o}= & \text { pipe slope, and } \\
S_{f w}= & \text { Energy grade line slope (Manning equation with fixed } n \text { ). }
\end{aligned}
$$

The inter-phase friction term, however, is calculated with an expression derived from the Darcy-Weisbach equation proposed by Arai and Yamamoto (2003):

$$
F_{i}=\lambda_{i} B \rho_{a i r}\left(u_{a}-u_{w}\right)\left|u_{a}-u_{w}\right| / 8
$$

where:

$$
\begin{aligned}
\lambda_{\mathrm{i}} & =\text { the Darcy friction coefficient, } \\
u & =\text { the local phase (air and water) flow velocities, and } \\
\rho_{\text {air }} & =\text { the local density of air. }
\end{aligned}
$$

The calculation of the surcharge head $h_{s}$ assumes elastic behaviour of the pipe walls, according to the Tube and Pipe Association formulation:

$$
h_{s}=\frac{a^{2}}{g} \frac{A-A_{\text {pipe }}}{A_{\text {pipe }}}
$$

where:

$$
a=\text { is the acoustic wavespeed. }
$$

The model by Vasconcelos and Wright (2009) applied a lumped air approach in that the air pressurization head $H_{\text {air }}$ was uniform in the extent of the air pocket. Air phase pressure in the whole pipeline was set as being equal to the computed value at the orifice location. More details on how this air pressure value is computed is presented in the Boundary Conditions subsection. 
However, for this work, air phase may also be computed in a discretized fashion, with the value of the air density and velocity varying point by point, and calculated with a specific mathematical model for the air phase. In this proposed model, the mathematical model selected to simulate the air phase pressure is the one dimensional, isothermal homogenous version of the Euler equations. This equation is applied to simulate unsteady gas flows, and may be expressed in primitive format as:

$$
\begin{gathered}
\partial \vec{U}_{a}+\frac{\partial \vec{F}_{a}}{\partial x}=S_{a} \\
\partial t \\
\vec{U}_{a}=\left[\begin{array}{c}
\rho_{a} \\
\rho_{a} u_{a}
\end{array}\right] \quad \vec{F}_{a}=\left[\begin{array}{c}
\rho_{a} u \\
\rho_{a} u_{a}^{2}+\alpha^{2} \rho
\end{array}\right] \quad S_{a}=\left[\begin{array}{c}
0 \\
-S_{f}-F_{i} / A
\end{array}\right]
\end{gathered}
$$

where:

$$
\begin{aligned}
& \rho_{\mathrm{a}}=\text { the gas density, } \\
& \alpha=\text { the speed of sound, assumed constant and equal to } \\
& \quad 343 \mathrm{~m} / \mathrm{s} \text {, and } \\
& a=\text { the air phase. }
\end{aligned}
$$

The friction force between air and the conduit walls slope is calculated as (Arai and Yamamoto, 2003):

$$
S f=\lambda_{i} P_{a} g \rho_{a} u_{a}\left|u_{a}\right| /\left(8 g A_{a}\right)
$$

where:

$$
\begin{aligned}
& P_{a}=\text { the air phase contact perimeter, and } \\
& A_{a}=\text { the cross sectional area. }
\end{aligned}
$$

An implicit hypothesis introduced in this air phase formulation is that the spatial gradient of the air flow within cross sectional area is assumed negligible. A formulation that accounts for variations on the cross sectional flow of the air, which in itself depends on the water flow calculation, is planned in the future.

With the updated values for the air density $\rho_{a}$, the air phase pressure head is calculated by means of the auxiliary relation for isothermal flows, in which $\gamma_{w}$ is the specific weight of the water (LeVeque, 1992):

$$
p=\alpha^{2} \rho_{a}=g \gamma_{w} h_{\text {air }}
$$

\subsubsection{Numerical Model}

\section{Internal Cells-Water Phase}

The proposed numerical model to calculate the water flow is constructed with the finite volume method, using a nonlinear numerical scheme based on the approximate Riemann solver proposed by Roe, as described by Macchione and Morelli (2003). The advantage of this nonlinear scheme is the ability to simu- 
late hydraulic bores with minimum numerical diffusion and oscillations, even with low Courant numbers. The conserved variables are updated with the expression:

$$
\begin{aligned}
U_{i}^{n+1}=U_{i}^{n} & -\frac{\Delta t}{2 \Delta x}\left[\left(F_{i+1}^{n}+F_{i}^{n}\right)-\sum_{j}\left|\overline{\lambda^{(j)}}\right|\left(\delta w^{(j)}\right)_{i+1 / 2} \bar{r}_{i+1 / 2}^{(j)}\right] \\
+ & \frac{\Delta t}{2 \Delta x}\left[\left(F_{i-1}^{n}+F_{i}^{n}\right)-\sum_{j}\left|\overline{\lambda^{(j)}}\right|\left(\delta w^{(j)}\right)_{i-1 / 2} \bar{r}_{i-1 / 2}^{(j)}\right]+\Delta t S_{i}^{n}
\end{aligned}
$$

where:

$$
n=\text { the time index. }
$$

The values of the approximate values of the eigenvalues $\lambda^{(j)}$ and the correspondent eigenvectors $\boldsymbol{r}^{(j)}$ for $A$ and $Q$ across the interface require first the computation of the Roe averages across each one of the interfaces $(i, i+1)$.

$$
\begin{aligned}
& \bar{A}_{i+1 / 2}=\sqrt{A_{i} A_{i+1 / 2}} \quad \bar{Q}_{i+1 / 2}=\frac{\sqrt{A_{i}} Q_{i+1}+\sqrt{A_{i+1}} Q_{i}}{\sqrt{A_{i}}+\sqrt{A_{i+1}}} \\
& \bar{c}_{i+1 / 2}=\sqrt{g \frac{I_{i+1}-I_{i}}{A_{i+1}-A_{i}}} \quad \text { if } A_{i} \neq A_{i+1} \quad \bar{c}_{i+1 / 2}=\sqrt{\frac{\frac{1}{2} g\left(A_{i+1}+A_{i}\right)}{\frac{1}{2}\left(B_{i+1}+B_{i}\right)}} \text { otherwise }
\end{aligned}
$$

With these values calculated, we now obtain the approximate eigenvalues and eigenvectors across interfaces $(i, i+1)$ with the equations:

$$
\begin{array}{ll}
\bar{\lambda}_{1}=\frac{\bar{Q}}{\bar{A}}+\bar{c} & \overline{\lambda_{2}}=\frac{\bar{Q}}{\bar{A}}-\bar{c} \\
\bar{r}^{(1)}=\frac{1}{2 c}\left[\begin{array}{lll}
1 & \bar{\lambda}_{1}
\end{array}\right]^{T} & \bar{r}^{(2)}=\frac{1}{2 c}\left[\begin{array}{ll}
1 & \bar{\lambda}_{2}
\end{array}\right]^{T}
\end{array}
$$

Finally, the strength of the wave crossing the cell interfaces $\delta_{w}$ can be obtained with the equations:

$$
\delta w^{(1)(2)}= \pm\left[\left(Q_{i+1}-Q_{i}\right)+\left(-\frac{\bar{Q}_{i+1 / 2}}{\bar{A}_{i+1 / 2}} \pm \bar{c}_{i+1 / 2}\right)\left(A_{i+1}-A_{i}\right)\right]
$$

\section{Internal Cells-Air Phase}

For the lumped air approach, the air phase is assumed uniform in the whole air pocket domain. Air velocity is not calculated for the whole pipeline, except at the air valve discharge point; shear forces due to air-water interactions are not calculated either. The update process for air phase is represented by the calcula- 
tion sequence below. It is important to notice that this approach requires no boundary conditions calculation.

1. Air pocket volume $V_{\text {air }, \text { old }}$ is calculated for that time step;

2. Based on the initial density $\rho_{\text {air,old }}$ for that time step, the discharged air mass $M_{a i r, d}$ during the time step $\Delta t$ is calculated using an orifice-type equation (equation 3.17) at BC;

3. After water phase calculation is complete for the time step, a new air pocket volume $V_{\text {air, new }}$ is obtained;

4. The new air density is calculated from the expression:

$$
\rho_{\text {new }}=\frac{\rho_{\text {old }} V_{\text {air }, \text { old }}-M_{\text {air }, d}}{V_{\text {air }, \text { new }}}
$$

5. Air velocity at the discharge point is calculated from the expression:

$$
u_{a}=\frac{M_{a i r, d}}{\rho_{a} A_{a} \Delta t}
$$

For the discretized air calculation, the Euler equations are solved with a finite volume solver, but instead of a more sophisticated nonlinear scheme, the simpler linear, first order accurate, Lax-Friedrich scheme is applied. Flow conditions for air are not anticipated to generate shocks, which explains this more simple approach. In future versions of the model the use of a nonlinear scheme to solve equation 3.5 (possibly HLL) will be considered.

The Courant number $(\mathrm{Cr})$ used in the simulation was 0.95 , and because air phase speed was larger than the assumed water acoustic wave speed, the local $\mathrm{Cr}$ for water phase was well below one, which was not an issue considering the use of a nonlinear scheme such as the Roe scheme used in this model.

\section{Source terms}

The source terms of the water phase were calculated according to Sanders and Bradford (2011), with the inclusion of the interface shear forces proposed by Arai and Yamamoto (2003). The resulting formulation preserves the stationarity of the solution and makes the calculation of the shear forces semi-implicit. This means that an approximation for the velocity of the $n$th time step is used for the calculation of the source term for the same time step, making it more precise for more abrupt velocity variations.

The equation for the source term $S_{0}$ is (Sanders and Bradford 2010), with $\rho$ being the water density: 


$$
\begin{aligned}
\mathrm{S}_{0}=-\frac{1}{\rho \Delta \Delta \mathrm{x}_{\mathrm{i}}}\left[\mathrm{I}\left(\mathrm{w}_{\text {level }_{\mathrm{i}}}+\frac{1}{2} \Delta \mathrm{w}_{\text {level }_{\mathrm{i}}}^{\prime}, \mathrm{h}_{\mathrm{s}_{\mathrm{i}}}+\frac{1}{2} \Delta \mathrm{h}_{\mathrm{s}_{\mathrm{i}}^{\prime}}^{\prime}\right)\right. \\
\left.-\mathrm{I}\left(\mathrm{w}_{\text {level }_{\mathrm{i}}}-\frac{1}{2} \Delta \mathrm{w}_{\text {level }_{\mathrm{i}}}^{\prime}, \mathrm{h}_{\mathrm{s}_{\mathrm{i}}}-\frac{1}{2} \Delta \mathrm{h}_{\mathrm{si}_{\mathrm{i}}}^{\prime}\right)\right]
\end{aligned}
$$

where:

$$
\begin{aligned}
w_{\text {level } i}= & \text { the water level in the pipe cell } i, \text { which will never be } \\
& \text { higher than the diameter of the pipe and the } I \text { function } \\
& \text { corresponds to the cross-sectional moment integrals } \\
& g A\left(h_{c}+h_{S}\right) .
\end{aligned}
$$

Considering that the source term may have an important role in the problem dynamics, an iterative approach is adopted to incorporate some of the source terms in the calculations. Firstly equations 3.1 and 3.2 are solved without the friction source term between pipe walls and water flow. The resulting approximated solution $\left(Q^{*}\right)$ is then applied in an iteration that incorporates the friction with the walls according to the formula presented by Sanders and Bradford (2011):

$$
Q_{i}^{n+1}=\frac{Q^{*}}{1+\Delta t\left(c_{D}\right)_{i}^{n} \frac{P_{i}^{n}\left|Q_{i}^{n}\right|}{\left(A_{i}^{n}\right)^{2}}}
$$

where:

$$
\begin{aligned}
& P_{i}=\text { the wetted perimeter at cell } i \text {, and } \\
& \mathrm{c}_{\mathrm{D}}=\mathrm{gn}_{\mathrm{m}}{ }^{2} \mathrm{R}_{\mathrm{h}, \mathrm{i}}{ }^{-1 / 3}
\end{aligned}
$$

where:

$$
\begin{aligned}
n_{m} & =\text { the Manning coefficient, and } \\
R_{h, i} & =\text { the hydraulic radius at cell } i .
\end{aligned}
$$

\section{Boundary Conditions-Water Phase}

At this point it is noted that the strategy is to solve for a water main with a single low point undergoing filling, with ventilation supplied at the downstream end by the downstream pipe (which is open to the atmosphere), while the upstream pipe ventilation is limited by an orifice located at the pipe's upstream end. More details on the proposed system geometry are presented in the following, and it is sketched in Figure 3.1 below (section 3.5 ).

At the upstream end of the model, an initial flow rate is supplied into the pipeline. The head in the reservoir upstream from the conduit is calculated and kept constant, so that this flow is feasible. However the inflow may decrease over time as result of air pressurization. The remaining conserved variable is calculated by assuming critical flow conditions at the upstream valve. This assumption may not be valid in cases when the discharge is supercritical at the valve. However, considering the geometrical complexity of the flow entrance, 
and the fact that the flow nature is best described by a three dimensional framework, this approximation may be sufficiently adequate for the purposes of the present comparative study.

At the downstream end of the downward slope section, a 4\% upward sloped pipeline is modeled applying a lumped inertia approach in its entire length. This modeling alternative was decided upon for two reasons: a decrease in the number of computational cells needed to be included in the simulations; and because it is assumed that the upward column is in contact with atmospheric air at the downstream end, therefore no air pocket entrapment is anticipated for this pipe during the filling process. After the downstream column flow parameters are updated, values of pressure and discharge are used as the boundary condition values for the water phase finite volume solver.

\section{Boundary Conditions-Air Phase (Discretized approach)}

The air phase simulation corresponds to a pocket that is expelled by an upstream moving bore, with a discharging orifice at the upstream end. The behaviour of the upstream orifice is represented by an equation that relates the discharge and the air density within the upstream computational cell (Zhou et al., 2002) considering that the orifice won't become chocked (thus having $\mathrm{Y}=1)$ :

$$
Q_{a i r}=C_{d} A_{0} \sqrt{2 g \rho_{w} / \rho_{a}\left(H^{*}-H_{a t m}^{*}\right)}
$$

where:

$$
\begin{aligned}
c_{d} & =0.65 \text { (assumed discharge coefficient) }, \\
A_{0} & =\text { the orifice area, } \\
H^{*} & =\text { the absolute air pressure head within the pipe, and } \\
H_{\text {atm }}^{*} & =\text { the absolute atmospheric pressure head }(10.3 \mathrm{~m} \text { at sea } \\
& \text { level). }
\end{aligned}
$$

By multiplying $Q_{\text {air }}$ by the density one obtains $M_{a i r, d}$ used in the lumped air calculation, and by dividing $M_{\text {air, }}$ by the cross sectional area of air, one obtains the second conserved variable for the air phase at the boundary condition. The other conserved variable is obtained with the relevant characteristic equation for the air phase assuming isothermal flow (Pulliam, 1994):

$$
u_{a, 1}^{n+1}=u_{a, 2}^{n}-\alpha\left(\log 10\left(\rho_{a, 2}^{n}\right)-\log 10\left(\rho_{a, 1}^{n}\right)\right)
$$

The iteration of equations 3.14 and 3.18 and the continuity equation within the upstream cell yields the values of $M_{a i r, d}$ and the two conserved variables at that location.

The downstream boundary condition is the moving pressurization bore. This bore can be approximated by a moving wall at the last computed air pocket cell 
plus one $(i=p l+1)$ and is calculated according as a reflective barrier using potential flow theory (Toro, 2001):

$$
\begin{aligned}
& \rho_{a, p l+1}^{n}=\rho_{a, p l}^{n} \\
& u_{a, p l+1}^{n}=-u_{a, p l}^{n}+2 w_{b o r e}
\end{aligned}
$$

where:

$$
w_{\text {bore }}=\text { the backward moving bore speed. }
$$

The bore's coordinate is obtained from the water phase calculation, and is obtained indirectly by tracking the location where the flow regime changes from free surface into pressurized. This tracking process is explicit and the interface location is updated as the pressurization front advances against the air phase. However, it does not involve in itself any calculation as it is required in mixed flow models that update the pressurization front using a shock fitting technique (e.g. Politano et al, 2007).

\subsection{Modeling Results}

To perform the comparison between the two modeling approaches to simulate the filling of water pipelines with limited ventilation, a two reach pipeline configuration with an intermediate low point was proposed. Pipeline overall length was $1500 \mathrm{~m}$, with the initial $1000 \mathrm{~m}$ in a $2 \%$ favorable slope, and a $600 \mathrm{~m}$ reach with a $4 \%$ adverse slope. Pipeline diameter is $1.0 \mathrm{~m}$, and Manning roughness was assumed to be 0.010 . The friction factor for air phase calculation was based on Manning roughness values. Figure 3.1 presents a sketch of the hypothetical pipeline.

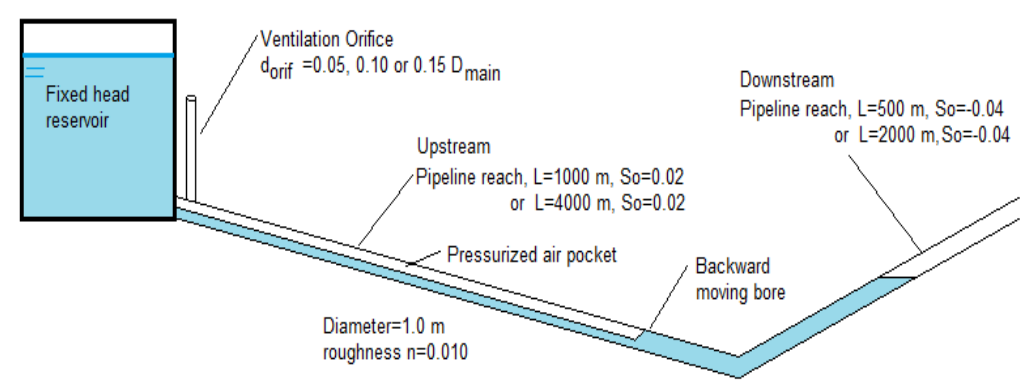

Figure 3.1 Sketch of the pipeline used in the numerical modeling comparison, with tested variables.

At the upstream end a fixed head reservoir provides an initial water inflow but does not permit air escape. The upstream reservoir head was chosen so that 
this initial flow rate would be in the desired range. There was no air escape through the upstream reservoir, and, depending on the degree of ventilation, air pressurization developed and decreased the inflow rate provided by it. Three ventilation sizes for the air valve were considered in terms of the pipe diameter D: $5 \%, 10 \%$ and $15 \%$ of $D$. Overall nine conditions were tested for each one of the modeling approaches, and the variables tested with respective tested range are presented in Table 3.1.

Table 3.1 Variables used in the numerical investigation.

\begin{tabular}{ll}
\hline \multicolumn{1}{c}{ Simulation parameter } & \multicolumn{1}{c}{ Range tested } \\
\hline$Q^{*}$ initial flow rates (normalized by $\left.\left(g D^{5}\right)^{0.5}\right)$ & $0.125,0.250$ and 0.500 \\
$d_{\text {orif }}^{*}$ Ventilation orifice diameter (normalized by $D$ ) & $0.05,0.10$ and 0.15 \\
\hline
\end{tabular}

\subsubsection{Pressure Predictions}

Figures $3.2\left(Q^{*}=0.250\right), 3.3\left(Q^{*}=0.500\right)$ and $3.4\left(Q^{*}=0.125\right)$ below present simulation results for the combination of the three orifice sizes used; the figures compare air phase pressure at the upstream end of the pipe and the water inflow rate into the system between the two modeling approaches. The time, pressure and flow rate scales are shared for each flow rate to illustrate the differences in magnitudes between the conditions of inflows and ventilation tested.

A description of the flow following the admission goes as follows. The flow is initiated at a constant rate (e.g. $Q=0.783 \mathrm{~m}^{3} / \mathrm{s}$ or $Q^{*}=0.250$ ), and the air phase pressure modeled is close to zero (atmospheric level), since there is a lot of ventilation supplied at the pipe downstream discharge. At some time later, the inflow front reaches the lowest point in the pipeline and fills the junction, blocking the ventilation and creating an air pocket at the upstream reach. This air is not initially subject to pressurization, and the pressure rises according to the inertial oscillations at the lowest point of the pipeline following the flow blockage at the low point, to some extent analogous to U-tube inertial mass oscillations. These oscillations are seen in the numerical predictions of both modeling approaches, but as the length of the lower column increases due to water mass accumulation, the oscillation period increases and the amplitude decreases. They are more pronounced for $Q^{*}=0.250$ than for the other flow rates tested, and are more persistent when the discretized modeling approach is used.

Air will remain pressurized during the filling event, and this pressurization causes a drop in the inflow admitted, since the water level at the upstream reservoir is kept constant by the assumed boundary condition. While air pressure will continue to rise, for the smallest ventilation tested, to significant levels, it becomes stable for orifices diameters of $10 \%$ and $15 \%$ of the water 
main diameter. Because flow rate decreases when air pressurization is more significant, the filling time may increase significantly (up to 25\%) when compared with a condition having a larger ventilation dimension.

As is apparent in the numerical results, predictions from both methods to simulate air phase are generally similar, particularly for the cases when the air pressurization is not significant (e.g. below $1 \mathrm{~m}$ water). For such cases, both the inflow rate and air pressurization become steady following U-tube type inertial oscillations.
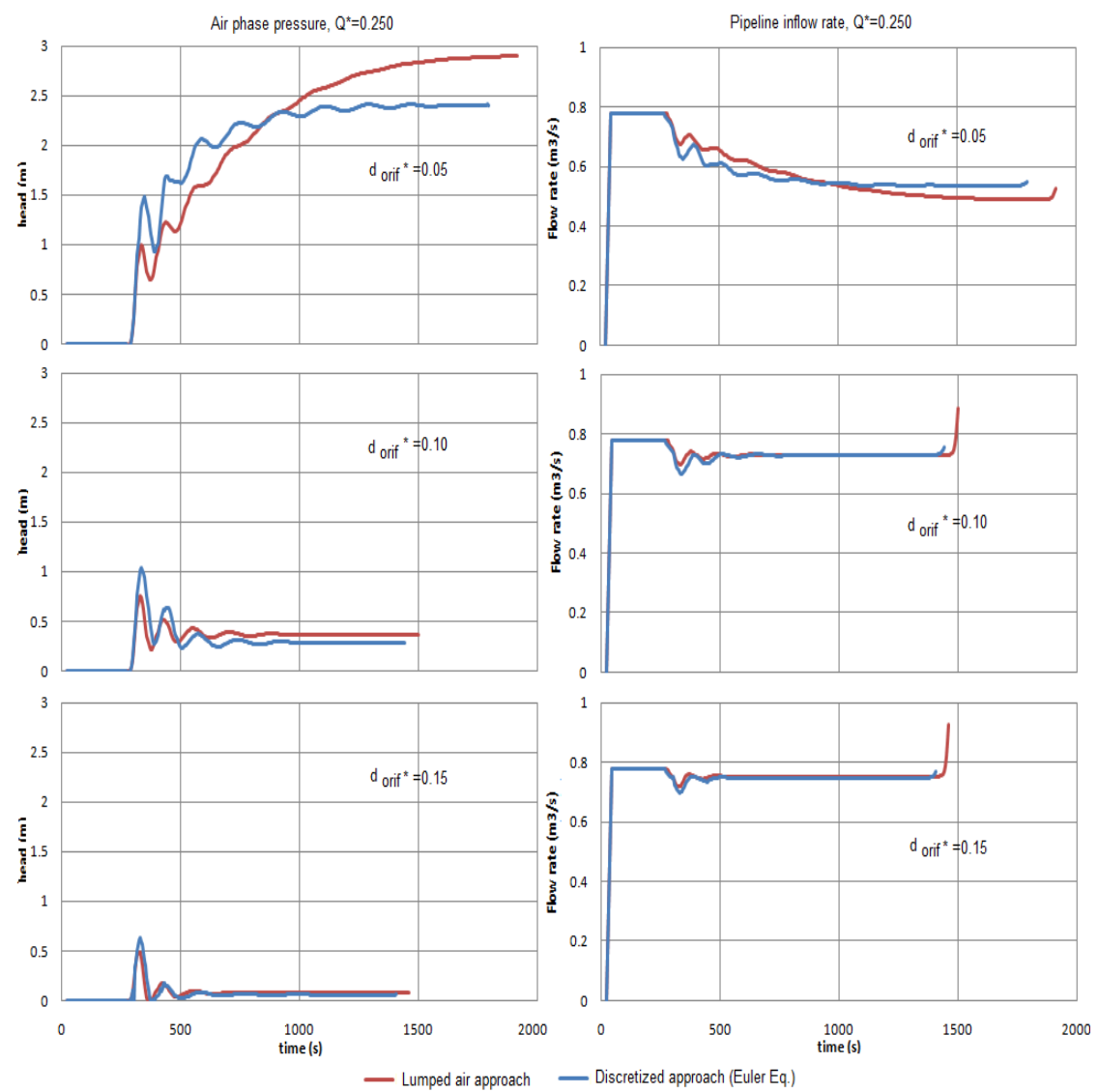

Figure 3.2 Comparison between two modeling approaches for the air pressure variation at the upstream end and inflow rate admitted for $Q^{*}=0.250$ and three ventilation sizes. 

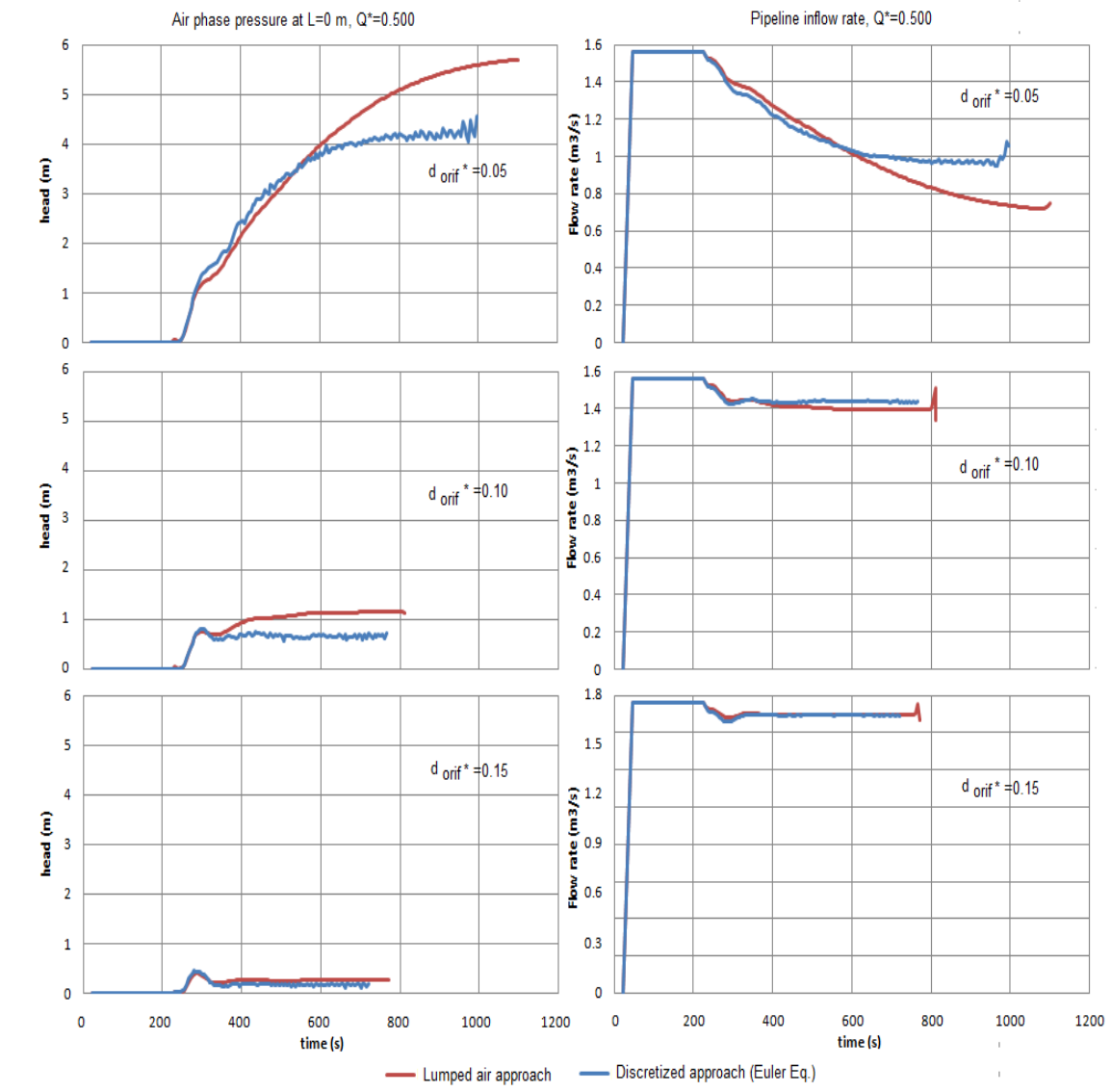

Figure 3.3 Comparison between two modeling approaches for the air pressure variation at the upstream end and inflow rate admitted for $Q^{*}=0.500$ and three ventilation sizes.

This behaviour is altered when the air pressurization becomes more significant, when the lumped air approach yields pressure results that may be up to $30 \%$ larger than the air pressure predicted by the discretized approach based on the Euler equation. For instance, for the case $Q^{*}=0.500$ and $d_{\text {orif }}{ }^{*}=0.05$ the air pressurization predicted by the lumped air approach is so much larger than that predicted by the Euler equations that even the filling time for the water main is $15 \%$ larger. 

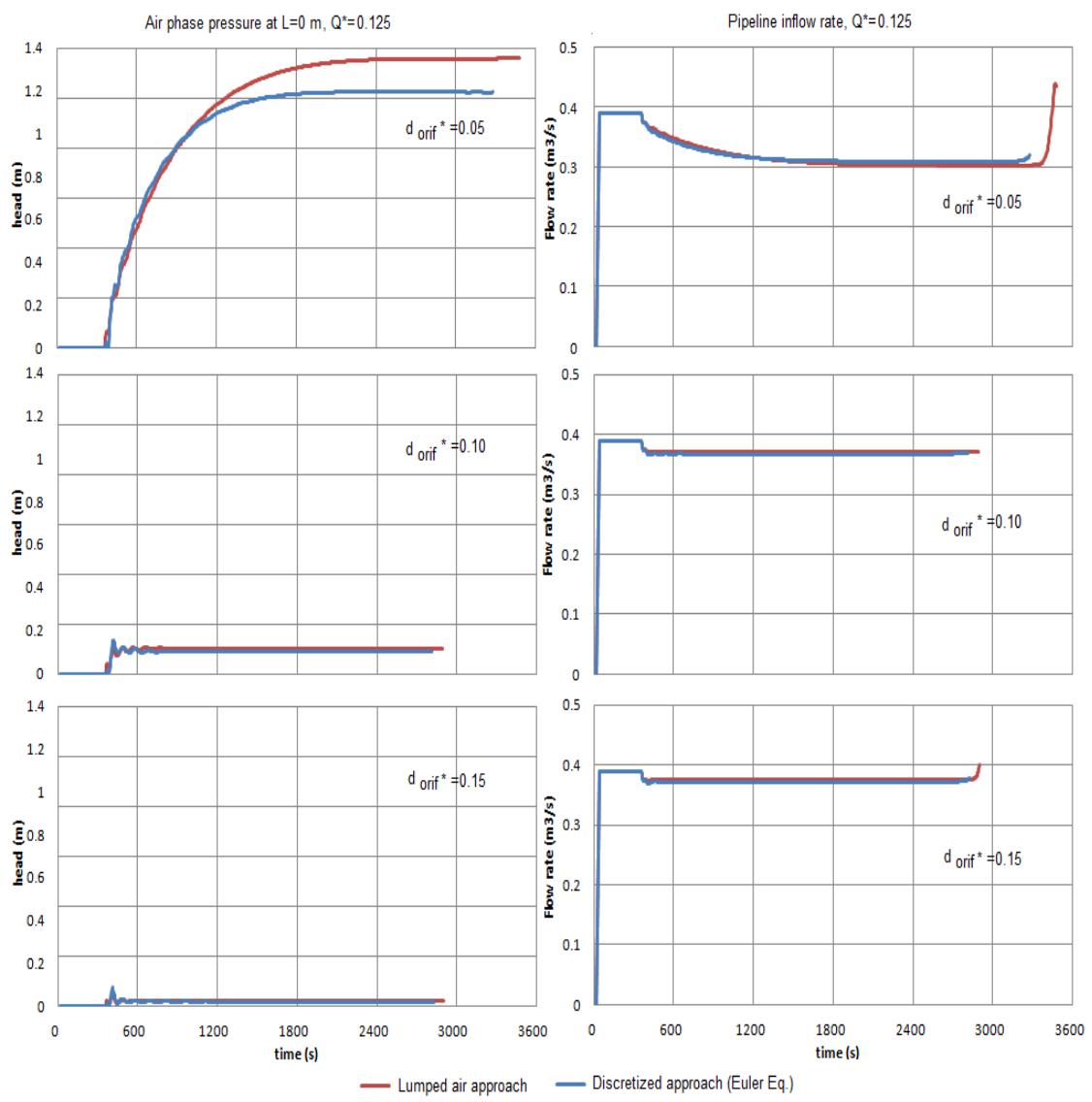

Figure 3.4 Comparison between two modeling approaches for the air pressure variation at the upstream end and inflow rate admitted for $Q^{*}=0.125$ and three ventilation sizes.

Simulation results presented in Figures 3.2, 3.3 and 3.4 indicate that the filling time is larger for conditions of smaller ventilations and flow rate, as expected, due to the increased air pressurization causing a decrease in the inflow discharge. A rapid filling condition should be linked to faster air flows towards the ventilation point. This is confirmed by the results for the Euler air-phase simulation model, presented in Figure 3.5. These results indicate stable air velocity values over $-0.3 \mathrm{~m} / \mathrm{s}$ for $Q^{*}=0.125,-0.8 \mathrm{~m} / \mathrm{s}$ for $Q^{*}=0.250$ and around $-2 \mathrm{~m} / \mathrm{s}$ for $Q^{*}=0.500$. Velocities are negative because the reference point adopted is that in which the positive direction is towards downstream and the air pocket moves upstream. Results from this figure, obtained with the 
discretized modeling approach indicate oscillations in this velocity (particularly for $Q^{*}=0.250$ ) during the inertial oscillation phase. The magnitudes of those oscillations seem exaggerated and are possibly caused by a limitation on how this phase is represented in the numerical code.

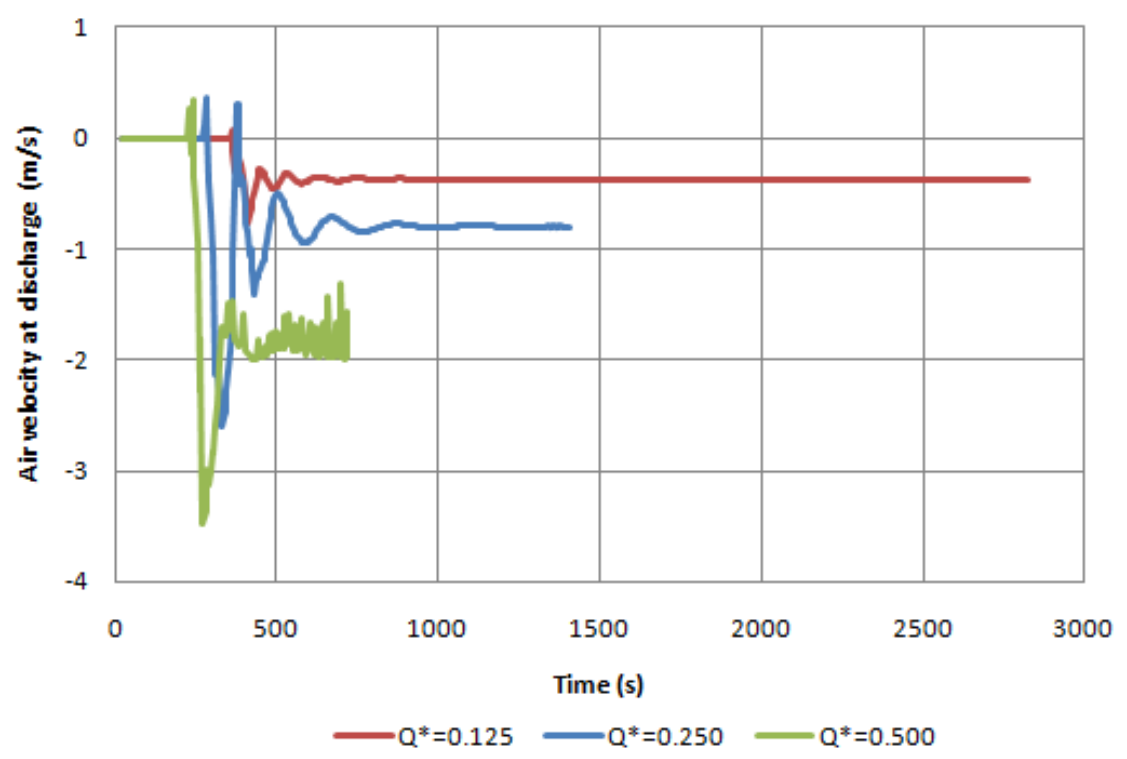

Figure 3.5 Comparison between air phase velocity at the upstream end predicted by the Euler equation model for all $Q^{*}$ tested and $d_{\text {orif }}{ }^{*}=0.15$.

Another result obtained from the discretized model is the prediction of the water pressure increase at the coordinate $X=500 \mathrm{~m}$ (halfway along the pipeline downslope reach) for each one of the tested flow rates. At that location, located at an elevation $10.0 \mathrm{~m}$ below the upstream end, the initial pressure is zero; then it grows a small amount corresponding to the water depth of the flow in free surface regime, filling the lower portions of the pipe. When the pressurization front arrives, pressure increases significantly and this increase is larger for larger air pressurization conditions (see Figure 3.6). Pressure continues to increase steadily until the pipeline is filled, and this increase is more pronounced for larger inflow rates. As is seen in Figures 3.2, 3.3 and 3.4, there are pressure oscillations or a spike on the inflow admission as the pipe becomes completely filled. It is speculated that the boundary conditions are not behaving as expected at this point in the simulation. 


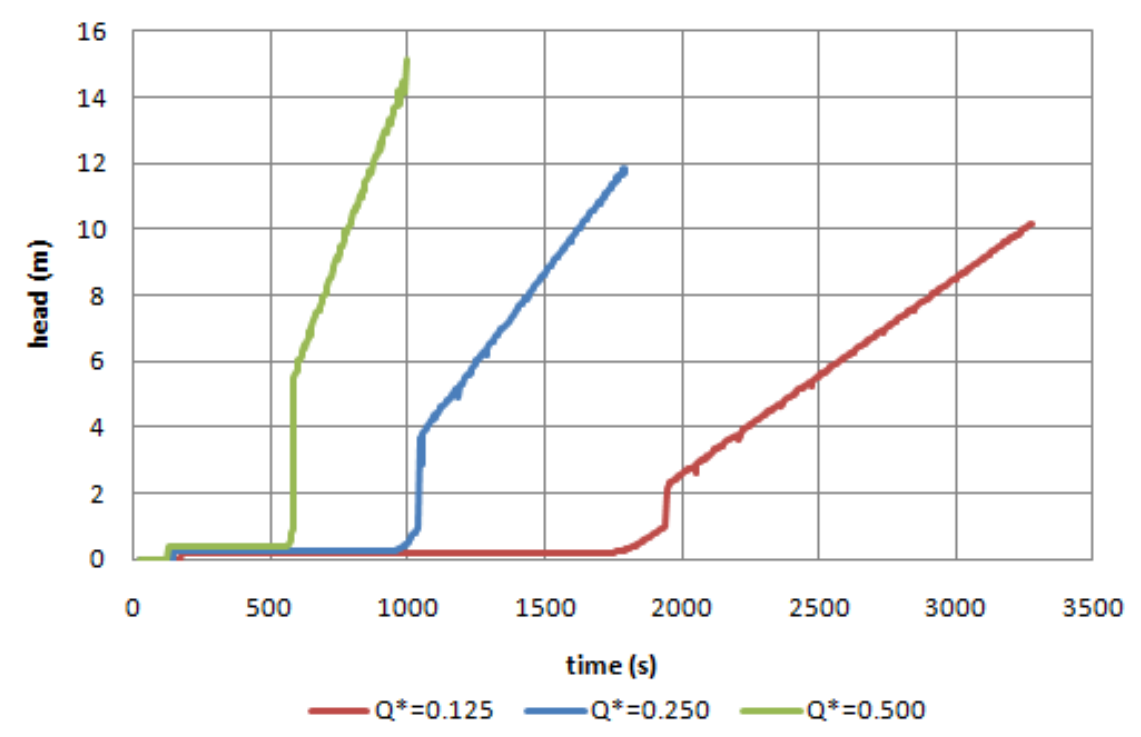

Figure 3.6 Comparison between water phase pressure at $X^{*}=X / L=0.50$ predicted by the Euler equation model for all $Q^{*}$ tested and $d_{\text {orif }}{ }^{*}=0.05$.

\subsection{General Comments and Future Work}

The importance of including air pressurization in problems involving the rapid filling of water pipelines is underscored by the numerical results presented by the present numerical methods. For the case of the system simulated, and depending on the combination of ventilation and inflow rate, air pressurization could be as large as $30 \%$ of the static head of the pipeline at the lowest elevation. Based on the actual pipeline filling event reported by Vasconcelos et al. (2009), the inflow $Q^{*}$ was 0.18 , and ventilation provided was closer to $d_{o r i f}{ }^{*}=0.15$. This combination is not likely to generate strong air pressurizations, provided that the air pocket is in contact with the ventilation point. But it points also to the fact that it may be possible to perform that type of filling with larger flow rates and still not generate excessively large pressure surges.

The differences between the lumped air approach and the discretized formulation appeared when the air pressurization head exceeded $1.0 \mathrm{~m}$, which also corresponds to the pipeline diameter. In such cases the lumped approach yielded larger air pressurization, and as a result smaller inflow rates into the pipeline and longer filling times. In general, computational time using the lumped approach was much smaller (about 56\%) than the computational time when using the Euler equations. 
Even though the results seem intuitive and both numerical models agree generally well between each other, it is very important to perform laboratory experiments to assess both numerical model approaches. While lumped air approaches yield more rapid predictions, if results from Euler equations models are more accurate then this modeling alternative should be adopted in future numerical developments to simulate pipeline filling events. These experiments are expected to occur very soon. Another change that will be implemented soon is to create a variation of the lumped air approach that also accounts for head losses in a steady air flow. If this is implemented and the results between the two modeling approaches becomes even closer, the simpler modeling alternative becomes even more attractive to be the alternative in the simulation of water main refilling operations.

\section{References}

Arai, K. and Yamamoto, K. (2003). Transient analysis of mixed free-surface-pressurized flows with modified slot model 1: Computational model and experiment. Proc. FEDSM03 4th ASME-JSME Joint Fluids Engrg. Conf. Honolulu, Hawaii, Paper 45266, ASME/JSME.

Cunge, J.A., Holly, F.M. and Verwey, A. (1980). Practical aspects of computational river hydraulics, Pitman, London

Guizani, M., J. Vasconcelos, S.J. Wright and K. Maalel. 2006. "Investigation of Rapid Filling of Empty Pipes." Journal of Water Management Modeling R225-20. doi: 10.14796/JWMM.R225-20.

Izquierdo, J., Fuertes, V.S., Cabrera, E., Iglesias, P.L. and Garcia-Serra, J. (1999). "Pipeline start-up with entrapped air.” J. Hydraul. Res., 37(5), 579-590. Leon, A.S., Ghidaoui, M.S., Schmidt, A.R., Garcia, M.H., (2009). "Application of Godunov-type schemes to transient mixed flows." J. Hydr. Res. 47(2), 147-156.

Leite, G.M. and J. Vasconcelos. 2011. "Numeric Modeling of Water Mains Filling Considering Air Pressurization." Journal of Water Management Modeling R241-01. doi: 10.14796/JWMM.R241-01.

LeVeque, R.J. (1992) Numerical methods for conservation laws Ed. Birkhauser and Verlag, $214 \mathrm{p}$.

Li, J. and McCorquodale, A. (1999) "Modeling mixed flow in storm sewers”. J. Hydr. Engrg., 125(11):1170-1180

Liou, C.P. and Hunt, W.A. (1996). "Filling of pipelines with undulating elevation profiles.” J. Hydr. Engng., 122 (10), 534-539.

Macchione, F.E. and Morelli, M.A. (2003). "Practical aspects in comparing the shockcapturing schemes for dam break problems". J. Hydr. Engng. 129(3), 187-195.

Martin C.S. (1976) "Entrapped air in pipelines”. In BHRA, editor, Proc. 2nd Int. Conf. Pressure Surges, Bedford, England.

Politano, M., Odgaard, A.J. and Klecan,W. (2007)."Case study: Numerical evaluation of hydraulic transients in a combined sewer overflow tunnel system". J. Hydr. Engng. 133(10), 1103-1110. 
Pulliam, T. H. (1994). "The Euler equations". Technical report, NASA Ames Research Center.

Sanders, B.F. and Bradford, S.F. (2011). "A network implementation of the twocomponent pressure approach for transient flow in storm sewers", J. Hydr. Engrg., 137 (2), 158-172.

Toro, E.F. (2001). Shock-Capturing Methods for Free-Surface Shallow Flows. John Wiley and Sons.

Vasconcelos, J.G. (2007) "Modelo matemático para simulação de enchimento de adutoras." Proc. $24^{\circ} \mathrm{CBES}$ - Brazilian Congress on Environmental Sanitation Engineering, ABES, Belo Horizonte, Brazil

Vasconcelos, J.G., Wright, S.J. and Roe, P.L. (2006). "Improved simulation of flow regime transition in sewers: The two-component pressure approach". J. Hydr. Engng. 132(6), 553-562.

Vasconcelos, J.G., Moraes, J.R.S. and Gebrim, D.V.B. (2009); "Field Measurements and Numerical Modeling of a Water Pipeline Filling Event” Proc. 33rd IAHR Congress, Vancouver, Canada

Vasconcelos, J.G., Wright, S.J. (2009). "Investigation of rapid filling of poorly ventilated stormwater storage tunnels", J. Hydr. Res. 47 (5), 547-558.

Zhou, F., Hicks, F.E. and Steffler P.M. (2002) "Transient flow in a rapidly filling horizontal pipe containing trapped air". J. Hydr. Engrg., 128(6):625-634. 
